\title{
LA MUJER ESPAÑOLA Y EL CONTROL DE NATALIDAD EN LOS COMIENZOS DEL SIGLO XX
}

\section{Raquel Alvarez Peláez}

Desde hace unos años me he dedicado al estudio de la elaboración, desarrollo y difusión de la teoría eugénica, de la eugenesia. Esta teoría, estas ideas, son en realidad poco conocidas aunque hay una cierta literatura anglosajona sobre el tema, tanto británica como norteamericana, por la incidencia que tuvieron ideas e instituciones eugénicas en estos dos países. En España no hubo instituciones de este tipo que fueran muy notorias, ni claras acciones prácticas a las que se les calificase de eugenésicas, pero sin embargo la eugenesia tuvo más influencia de la que suponemos en las ideas relacionadas con los problemas de decadencia y degeneración de la raza española, del pueblo español, que se plantearon muy agudamente a comienzos de siglo; y la tuvo también en relación con la importante, tremenda cuestión, de la enorme mortalidad infantil que existía en nuestro país. Este acuciante problema llevó a Marañón a expresar, en 1920, lo siguiente:

Una ley aterradora: la fecundidad de las madres está en relación directa con la mortalidad de los hijos. Mas preguntadles ahora cuantos hijos viven de los que dieron a luz, y os aseguro que vuestro optimismo se trocará en terror, porque de esoshijos, engendrados en pleno trabajo, paridos con tanto dolor, amamantados exprimiendo el organismo exhausto, no quedan ni la mitad, muchas veces menos, quizá sólo uno o ninguno. No creaís que exagero. Voy a leeros una estadística macabra que os lo probará. Se refiere a mujeres de todas las provincias de España, excepto Cataluña, Baleares y Canarias. Todas estas 
mujeres, escogidas al azar, pertenecen al proletariado. Todas han terminado ya su ciclo sexual; los datos que proporcionan son ya por lo tanto, invariables. Estos datos nos dicen:

Que un 28 por 100 de dichas mujeres había tenido más de ocho hijos: diez, doce o más; con frecuencia dieciséis o diecisiete; en dos casos hasta veinte. Esta proporción de mujeres de elevada fecundidad, es realmente extraordinaria, muy superior a la de los países más cultos de Europa. Pero de los 473 hijos de estas mujeres fecundísimas, han muerto 382. Fijaos bien: 473 nacimientos y 382 muertos. Es decir una mortalidad que supera el 80 por 100 (..). Un 54 por 100 de las mujeres examinadas habían tenido un número de hijos oscilando entre 1 y 7 . La mortalidad de este grupo, siendo también elevadísima, es menor que la del grupo anterior: un 65 por 100 . Si de esta serie de mujeres aislamos todavía el núcleo de las que han sido madres de un número, que pudiéramos llamar normal de hijos -3 a $5-$, veremos que la cifra de mortalidad desciende hasta el 59 por 100 . ¡Ved, pues, qué tremendo, qué estéril esfuerzo el de nuestras pobres mujeres! ¡Qué número tan grande de hijos! (...) Pero casi todos estos hijos numerosos desaparecen antes de ser hombres o mujeres útiles, porque la madre no ha podido engendrarlos fuertes, ni cuidarles luego su debilidad o sus enfermedades; porque la escasez del hogar no alcanza a alimentarles suficientemente; porque el Estado, en fin, no suple con una acción protectora la miseria familiar. ¿Qué dirán ahora, ante estas cifras abrumadoras, nuestros sociólogos entusiastas? El neomaltusianismo más depravado no ha logrado, en el país que se considere más inmoral, ni acercarse remotamente a los estragos que produce entre nosotros la miseria y la ignorancia. ¿Cuál será por este camino el porvenir de la raza? Porque aunque las estadísticas generales, por incluir las familias ricas, en las que la mortalidad infantil es mucho menor, dan atenuadas estas cifras tremendas, ya en el año pasado, a pesar de todo, la mortalidad global de España (18.147) ha superado a la natalidad (16.309)» (1).

La eugenesia, nacida en la Inglaterra victoriana, institucionalizada en Gran Bretaña a comienzos del siglo Xx, ofrecía una teoría «científica» que tendía a buscar soluciones a la preocupación - que no era sólo española - por el estado de la población, y no sólo por su cantidad, sino por su calidad, que en nuestro caso era un problema fundamental, como hemos visto en lo expresado por Marañón. Se procreaban niños suficientes y más que suficientes, pero se morían. El estado físico de nuestros hombres y mujeres de las llamadas clases bajas era muy malo, y existían enfermedades que eran verdaderas plagas: sífilis y tuberculosis sobre todo. 
El estudio del desarrollo y penetración de la eugenesia en España me ha permitido, por sus inevitables conexiones con tal problema, tomar contacto también con la cuestión del control de natalidad o control de la procreación. De hecho la eugenesia es una de las teorías a las que se adjudicaba el preconizar las técnicas del control de natalidad, cosa en sí falsa, como veremos a continuación.

Se han confundido frecuentemente los contenidos de diversas teorías y posiciones con respecto al control de natalidad, y fundamentalmente creo que no se han comprendido las diferencias que existen en el contenido de proposiciones como la de Malthus, a finales del siglo XVIII, el Neomalthusianismo y el Birth Control de mediados de siglo XIX y la eugenesia, nacida en la segunda mitad del siglo XIX pero puesta en funcionamiento a comienzos del XX.

\section{Malthusianismo}

Comenzaremos, para seguir un orden cronológico que tiene su sentido histórico inevitable, aunque no podamos entrar en él, hablando de Malthus.

Como bien comprendía Hildegart, _ joven abogada española de los años treinta y secretaria en esos años de la sección española de la Liga para la Reforma Sexual sobre bases científicas, posiblemente de las pocas personas entre los españoles, y ni que decir tiene, mujeres, que había estudiado el tema del control de natalidad en profundidad (2)la teoría de Malthus había sido una teoría económica. En ella su autor exponía que Gran Bretaña podía producir una determinada cantidad de alimento, que esto permitía alimentar correctamente a un cierto número de individuos, y que por lo tanto, cuantos más individuos peor el reparto, más pequeña la porción. De ahí se deducía que era aconsejable que la población aumentara lo menos posible. Por otra parte, los factores que hacían que la población no aumentara aún más eran el vicio y la miseria, que permitían que los peores murieran (de aquí surgirá la inspiración de Darwin, y también la idea de Spencer de la «lucha por la vida»). Estas dos conclusiones, necesidad de controlar el aumento de población y mecanismos naturales de control en la sociedad, le llevaron a defender dos actitudes prácticas: proponer que los matrimonios se retardaran, o en todo caso, la abstinencia, para que disminuyera la fertilidad; oponerse terminantemente a la Poor Law, la ley inglesa que protegía a los pobres, en esa época en general distribuidos 
por pequeños pueblos o viviendo en el campo, pues no había todavía grandes aglomeraciones urbanas. La beneficencia, el dar de comer a los pobres y miserables y el curar sus enfermedades era, para Malthus, socialmente negativo, pues mantenía una sobrepoblación que nunca podría salir de la miseria puesto que la producción de alimentos nunca sería suficiente (3).

Esto es en esencia lo que mantuvo Malthus, que nunca se refirió, ni su condición de religioso lo hubiera permitido, al control de natalidad por otros medios que los ya indicados.

\section{«Birth Control»y Neomalthusianismo}

En los mismos años en que la vida de Malthus tocaba casi a su fin, en la década de los años veinte del siglo XIX, comienza a surgir en Inglaterra la propaganda a favor del uso de métodos de control de natalidad. En este país muchos de los métodos se conocían ya desde el siglo XVIII, por lo que no parece haber sido realmente una novedad para la gente. Lo que sí era una novedad era que estos métodos se publicaran y se aconsejaran, además, como forma de aumentar la libertad individual, de evitar el aborto y el infanticidio, y de colaborar para que se pudiera criar mejor a los hijos que se tuvieran. Esta fue la actitud que difundían los partidarios del Birth Control (4).

Los malthusianos se oponían a esta actitud que tendía, como decía el mismo Malthus refiriéndose a Condorcet, a «destruir la virtud y pureza de las costumbres» (5). Los únicos que apoyaron sin problemas a los propagandistas del Birth control fueron los Benthamitas, los «utilitarios» (6), que no se planteaban problemas religiosos que les hicieran cuestionarse la actitud de control de la natalidad. Así los Mill fueron también propagandistas de las necesidad de tal control, como también Richard Dale Owen, hijo de Robert Owen.

Se da como fecha de origen del movimiento del Birth control el año 1823, cuando Francis Place distribuye panfletos de propaganda en los distritos obreros de Londres. Explicaba en ellos el uso de la esponja y el coitus interruptus, o la "retirada», como le llamaban los ingleses. Folletos posteriores explicaban también el uso del preservativo, que había surgido en un principio como protección frente a las enfermedades venéreas, (protección de los hombres, claro.)

Las ideas de los propagandistas del Birth control no tenían, pues, nada que ver con las ideas de Malthus. Y se oponían a su aspecto más 
esencial, puesto que ofrecían una alternativa a la gente frente a la abstinencia, al matrimonio tardío y también, según preconizaban, frente a la miseria. Sin embargo la mayoría de la gente confundía ambas doctrinas porque las dos buscaban la disminución, o mejor dicho el control del crecimiento de la población. Los movimientos obreros se oponían al Birth control porque lo consideraban como un movimiento a favor de la burguesía, que tendía a eliminar o mermar a la clase obrera (Actitud semejante mantendrán algunos sectores anarquistas de España en las primeras décadas del siglo veinte). Por otra parte, la moral tradicional señalaba que esa actitud de control de natalidad era ir contra Dios y contra la Naturaleza, actitud, pues, inaceptable. Y, como veremos más adelante, la postura de los médicos era también negativa hacia la posibilidad de contener y autocontrolar la procreación.

La propaganda a favor del control de la natalidad tuvo su auge en las décadas de los años veinte y treinta. En esta última década fue cuando algunos pensadores, como John Stuart Mill, integraron la contracepción dentro de un programa de reforma social, pero esta fue una postura minoritaria. De hecho, los iniciadores de la campaña del Birth control fueron tomando, con el tiempo, una postura cada vez más conservadora desde el punto de vista político.

\section{Neomalthusianismo}

A partir de los años cincuenta del siglo XIX, la propaganda del control de natalidad estaba prácticamente dominada por conservadores, personalidades tales como George Drysdale y Charles Bradlaugh. Sus ideas conducían a luchas contra algo que se hacía cada vez más evidente: el que, a pesar del control y freno que pudieran establecer la miseria y el vicio, las clases trabajadoras, y no sólo éstas, sino los paupérrimos habitantes de los ya existentes suburbios, se reproducían más que las clases medias y altas. Estas preocupación, que se hacía más acuciante y evidente a medida que se desarrollaban las estadísticas de población (7), demostraba al mismo tiempo el estado de deterioro de esa población. Estado de deterioro que se hizo aún más evidente cuando el Estado tuvo necesidad de reclutar soldados para la guerra de Crimea (1855) y también durante el desarrollo de ésta, a la vista de la enorme mortalidad que se produjo por las malas condiciones higiénicas de viviendas y enfermerías (8). No se planteaba ya, como había hecho a fines del dieciocho Malthus, la preocupación por la cantidad de población, que en muchos casos como en el de la guerra a gran escala podía 
ser positiva, y que además la Revolución Industrial podía llegar incluso a conseguir alimentar. Se trataba ahora de un problema de calidad, de buen estado físico y mental del pueblo. En esta época comienza además la gestación de la teoría eugénica como teoría que busca justamente eso, mejorar la calidad de la raza. Pero esta doctrina no cobrará impulso hasta comienzos del siglo XX.

A partir de los años cincuenta comienza, sin embargo, lo que podríamos llamar movimiento Neomalthusiano, que se hace firme en 1877 con la fundación de la Liga Malthusiana. Nos encontramos con un movimiento que propicia el control de nacimientos, pero que no hace propaganda abierta de los métodos. Lo que intenta es que la necesidad del control de natalidad sea asumida por los médicos. Como veremos cuando nos refiramos específicamente a la actitud de éstos, los médicos, la profesión o corporación médica siempre se negó a asumir tal cosa y tomó en general la postura menos comprometida, la del silencio.

A pesar de la actitud claramente conservadora de la Liga Malthusiana, absolutamente opuesta a los movimientos socialistas que surgían en esos años, y a toda idea de lucha de clases, no consiguieron hacer respetable el control de nacimientos. Quisieran o no, su planteamiento cuestionaba las relaciones sexuales existentes y la moral victoriana. Por otra parte, los neomalthusianos trataron muy seriamente los derechos de la mujer.

A pesar de que los movimientos feministas luchaban sobre todo por una serie de derechos políticos, había una inevitable relación entre ellos y la idea de planificación familiar. Hay evidencias que demuestran que las mujeres respondían bien a la propaganda de los birth controlers. Pero muy posiblemente estas actitudes no se manifestaban más que a un nivel casero, o más aún, sólo a nivel de las mujeres. Hay también evidencias que demuestran que muchas mujeres utilizaban métodos anticonceptivos - duchas vaginales, diafragmas, esponjas-incluso sin que lo supieran sus maridos. Parece claro, además, que la disminución progresiva de natalidad que se produjo a lo largo de la segunda mitad del siglo XIX en las clases medias y altas respondía a una toma de medidas voluntarias.

De todas maneras, el intento de la Liga Malthusiana de ganarse a los trabajadores, las mujeres y los médicos fue un continuo fracaso. Y debemos señalar desde ya que lo mismo y más sucedió durante el primer tercio del siglo XX en España. Evidentemente también porque el movimiento neomalthusiano fue muy pequeño y muy localizado, y por otras razones que veremos al tratar de la situación española. 
Hacia finales del siglo XIX surge en Francia una ramificación neomalthusiana de carácter libertario, la Liga de Regeneración Humana fundada por Paul Robin, que pasaría a España de la mano de Ferrer i Guardia y de Mateo Morral, que fundarían la liga correspondiente en Barcelona, y una revista y editorial difusora de libros y panfletos de propaganda, Salud y Fuerza (9). Los anarquistas, sin embargo, se oponían en general al control de natalidad, pues consideraban que todo el mundo tenía derecho a tener hijos, y que lo que se debía solucionar era que todo el mundo pudiera tener unos hijos sanos y fuertes, bien alimentados y educados. El control de natalidad era pues una actitud que favorecía a la burguesía. Así lo manifestaban en La Revista Blanca Soledad Gustavo y Federico Urales cuando comentaban la aparición de la revista Salud y Fuerza (10).

Los neomalthusianos ingleses se desesperaban, pues como decían en su periódico The Malthusian, los obreros se inclinaban por el socialismo, las mujeres se dedicaban a luchar a favor del sufragio femenino y los médicos o no se pronunciaban sobre el problema de la procreación o se pasaban a la eugenesia. De esta visión de la cuestión hablaremos ahora, de la eugenesia.

\section{Eugenesia}

La doctrina eugnénica fue elaborada por Francis Galton - primo de Charles Darwin y prototipo de lo que se ha dado en llamar un "victoriano»-, en los años sesenta del siglo XIX. La definió como tal doctrina y la bautizó como eugenesia en 1883, en su libro Inquiries into Human Faculty (11). La doctrina eugénica no cobró auge, ni se popularizó, sin embargo, hasta que en 1908 se funda la Eugenics Education Society, formada por preeminentes profesionales, gran parte de ellos médicos, que comenzó una gran campaña de difusión de la eugenesia a través de cursos, conferencias y publicaciones. En 1912 se lanza a la conquista del medio internacional a través del Primer Congreso Internacional de Eugenesia, punto de partida de varias sociedades eugénicas europeas, al que acudieron conocidas figuras como Sergi o Magnan. También hubo una representación española, la del médico catalán Valentí y Vivó, al que se consideró representante de la Universidad de Barcelona (12).

La doctrina eugénica establecía que todas las características humanas, físicas, mentales y morales eran producto de la herencia. Que esta herencia era absolutamente determinante y que la educación y el cam- 
bio ambiental sólo eran factores favorecedores o no del desarrollo de esas características heredadas, pero no podían cambiar una situación de degeneración o decadencia de una raza. Por lo tanto, la única manera de superar el problema - que se sentía como acuciante no sólo en Inglaterra, sino en la mayoría de países incluida España (13) - de la progresiva degeneración de la raza, causada fundamentalmente porque las clases peor dotadas (las clases bajas) se reproducían más y más, y las mejor dotadas (fundamentalmente las clases medias) cada vez menos, era actuar controlando la reproducción mediante una «selección artificial» semejante a la que realizaban los criadores de animales para mejorar sus razas. Era una forma de ayudar a la «selección natural" a superar los obstáculos que la sociedad ponía a su acción por medio de la sanidad, beneficencia, etc. La eugenesia preconiza, entonces, el control de los matrimonios, pero no como había planteado Malthus, buscando una disminución en la cantidad de hijos, sino buscando una mejor calidad a través de la selección de los padres procreadores. Había, pues, que establecer un control de los matrimonios. Estudiar a los posibles cónyuges en todos los aspectos - estado de salud actual, historial patológico y no patológico- personal y, muy importante, familiar. Y hacer que las parejas bien dotadas e idóneas para procrear tuvieran una ayuda del Estado (14). El talento era, claro, un factor fundamental en la selección.

Lo cierto es que este tipo de acciones, tendentes al control de los matrimonios positivos para la raza, era muy difícil de realizar y, además, posiblemente la gente se resistiría a semejante control sobre su vida privada. Se comenzaron a proponer entonces, con más fuerza, las medidas de «eugenesia negativa», llamadas así en contraste con las otras a las que se señalaba como medidas de «eugenesia positiva». Las medidas de eugenesia negativa consistían fundamentalmente en impedir que los llamados débiles mentales, los locos, epilépticos o los enfermos de tuberculosis o sífilis; que los criminales, pobres de solemnidad o vagabundos con poco apego al trabajo, tuvieran hijos. La propuesta de Galton para solucionar esta situación era el confinamiento. Sin embargo, sus seguidores norteamericanos pusieron rápidamente en marcha, desde los primeros años del siglo veinte, una medida mucho más práctica, más radical y menos costosa: la esterilización forzosa, practicada a partir de la decisión positiva de un juez ante el que se exponía el caso. Estas medidas, junto a la del establecimiento de un certificado médico prematrimonial, se convirtieron en las dos actitudes prácticas más realizables de la eugenesia. 
La eugenesia no planteó de ninguna manera, hasta muy avanzado el siglo XX, la anticoncepción. Y cuando la Sociedad Eugénica admitió a los Drysdale, defensores del control de natalidad, lo hizo con muchas reticencias y muy recatadamente, sin dar mayor publicidad al asunto, que aceptó casi por imposición de las circunstancias. No recuerdo ninguna publicación de la Sociedad Eugénica en que se hiciera propaganda, y ni siquiera se mencionara la anticoncepción, aunque sí siempre estuvieron a favor y difundieron la educación sexual de los jóvenes.

Tenemos, pues, definidas, las doctrinas que de alguna manera se relacionaron desde un principio con el control de natalidad. Podemos decir después de lo expuesto que de hecho los partidarios del Birth control fueron muy pocos, en general grupos minoritarios enfrentados con la sociedad. Lo que no quiere decir que no se utilizaran métodos anticonceptivos, y que no hubiera, posiblemente, una abundante transmisión oral de información en tal sentido.

Entraremos ahora a considerar la situación de la mujer en la sociedad, y que factores condicionan su receptividad y posibilidad de luchar por problemas que le son fundamentales para su desarrollo.

\section{El contexto y el concepto de mujer}

La población, la producción de seres humanos, ha sido y sigue siendo una cuestión de enorme importancia. Pensemos que el volumen de población no debe sólo medirse en términos absolutos, sino también en valores relativos a la extensión del medio habitable, a la capacidad de éste para producir alimentos - sea naturalmente, espontáneamente o por actividad del hombre- y a sus condiciones climáticas, etc. La cuestión de la población se ha planteado frecuentemente como problema, tanto fuera por escasez como por superabundancia de personas. En general, parece claro que todos o muchos de los pueblos han utilizado alguna forma de regulación de la población, como han comprobado y estudiado muchos antropólogos. Tanto fuera la lactancia prolongada, como forma de espaciar los embarazos, el infanticidio (la mayor parte de las veces de las niñas), como cualquier otro método, estaba en general integrado a la cultura o a la religión del pueblo que lo utilizaba. El desarrollo de la civilización occidental, el crecimiento de sus diferentes sectores, su complejidad, cuestión que no podemos detallar aquí, llegó a crear una situación de graves contradicciones entre deter- 
minadas necesidades prácticas y las múltiples soluciones que a esas necesidades prácticas se les puede dar. La población, el número de seres, ha jugado siempre un papel importante en esos mecanismos y en esa búsqueda de posibles soluciones a la crisis. Las religiones, como sistemas morales o éticos que han conformado el sistema social, imponen una serie de condicionantes que pueden favorecer a unas u otras de las posibles soluciones que se pueden dar a una situación, por ejemplo, de superpoblación.

Lo importante para nosotros en este caso es que la mujer, como productora directa de ese bien tan controvertido pero siempre importante, el ser humano, está en el centro de la cuestión. La mujer es el ser reproductor, pero además se convierte, no violentamente, sino por su autoconvencimiento y por la realidad biológica de su situación de reproductora, y por presión del medio, de la cultura, de la ideología y de la religión -en definitiva por el sistema a través del cual se comprende y se observa la realidad- se convierte, decía, en casi (o sin casi) exclusivamente eso, la madre por excelencia. Más importante que su vida como ser, como animal humano, es su papel de madre; de hembra procreadora, en fin, puesto que lo de «madre» es un montaje cultural con múltiples connotaciones más o menos alejadas de la realidad.

El cristianismo contribuyó enormemente a enaltecer tal situación de ser madre antes que persona. El hombre, Dios, Jesucristo, es el ser central. Pero la madre es un elemento fundamental, incluso despreciando en cierta medida y posponiendo la figura del padre. Es, pues, el hombre, como Dios o su hijo lo que es importante, crucial. Pero es la madre, pero no la mujer, lo que es importante y crucial. Al despojarle de la pérdida de virginidad se le ha despojado, además, de su realidad de mujer, que se siente como contaminante. La mujer es pecado, la madre no. La cultura española está, y estaba mucho más, total y profundamente impregnada de esta concepción. Es algo que debemos tener absolutamente en cuenta si queremos comprender no sólo la postura de la mujer española frente a los métodos anticonceptivos, sino también la del hombre. Lo que se produce en España va más allá de un problema de derechas e izquierdas, de socialistas, liberales o conservadores desde un punto de vista político. Es algo más profundo, es una autocensura cultural y moral creada, no por una religión, sino por una determinada concepción de la religión. A partir de aquella primera concepción judía y cristiana de la mujer, se produjeron, dentro de una misma postura indudablemente machista, desarrollos de las religiones con diversos matices, según las circunstancias históricas en las que se de- 
senvolvieron. La religión cristiana formó parte indisoluble de la sociedad inglesa, y los ingleses han sido religiosos. Pero probablemente la diversidad de grupos que aparecen, las distintas concepciones en cuanto a la organización de la Iglesia que se desarrollaron, la aproximación directa al cristianismo de muchos grupos, y quieras que no, la menor fuerza de la jerarquía eclesiástica que se impone a partir de la edad moderna, además de las múltiples controversias que con tal circunstancia pudieron mantenerse, rompieron con la fuerza monolítica de una posición única y una concepción única del sistema y del sentimiento religioso. En nuestro país, después del concilio de Trento y gracias al desarrollo de una postura contrarreformista a ultranza, sucedió exactamente lo contrario.

Pensemos que muchos de los defensores del Birth control de la Liga Malthusiana no sólo eran conservadores políticamente, sino también profundamente religiosos. Y también es cierto que podían ser conservadores y no religiosos. En fin, que las opciones y matices eran mucho más variados y no había unos esquemas tan rígidos que indicaran, ya antes de cualquier discusión de un problema, en este caso del control de natalidad, la postura que obligatoriamente, para ser consecuente, se debía tomar. En España todo se convertía en precepto religioso. Esto no quiere decir que ya en el siglo XX no se produjeran casos semejantes a los de Inglaterra en nuestro país. Médicos, abogados e incluso 'eólogos -como Torrubiano Ripoll- que a pesar de sus ideas polít icas y religiosas consideraban razonables y en absoluto opuestas a la religión católica, una serie de medidas que la famosa «cuestión social» española, el estado de deterioro físico de su población, hacían ineludibles.

Pero debemos de partir de la base, pienso, de que la moral católica indicaba que el fin del matrimonio era tener hijos, era la procreación, y que todo otro aspecto de la relación era pecado: el placer de la relación sexual, por ejemplo; el placer de la relación personal, humana, no procreativa. Y la mujer española estaba profundamente convencida de la importancia de su papel de madre procreadora por encima de cualquier otro. En la literatura se ha repetido hasta la saciedad la actuación pecaminosa de mujeres que han preferido su relación con un hombre, demostrando con más o menos crudeza la perversión de tales sentimientos, frente a la nobleza de las que han preferido su papel de madres aunque fuera jugando un papel que humanamente podía considerarse absolutamente humillante. El haber planteado tal situación como alternativa, el que llegara a asumirse así, como alternativa, por las pro- 
pias mujeres, fue posiblemente una de las situaciones más negativas, dolorosas y castrantes de la estructuración de la sociedad española, de sus esquemas culturales.

Nadie puede renegar de su papel de madre porque sí. Y es difícil, cuando todas las circunstancias hacen que ser madre sea - cuando está dentro de la norma- algo que da categoría propia y es un papel absolutamente ensalzado, y que no serlo sea algo tremendamente vacío, que hace prácticamente inexistente a un ser socialmente y que sólo provoca la conmiseración; es difícil, creo, tomar actitudes que aparenten estar en contra de tener hijos o en contra del papel absoluto de madre.

Se contraponen, además, y me estoy refiriendo al siglo veinte, el trabajo de la mujer a la buena crianza de los hijos, a la relación correcta con el marido, a la buena marcha de la familia toda, que es en realidad el trabajo ensalzado, el elevado trabajo de la mujer, para el que ha sido biológicamente destinada. Existe toda una presión culpabilizante que endurece tremendamente cualquier postura de lucha contra esta concepción.

Debo decir que, aparte la cuestión religiosa que conforma la moral y la ética social, el mecanismo de situar a la mujer en un papel casi único, de manera que cualquier otro haya tenido que conseguirse con lucha, es general en toda sociedad. Sin embargo, como ya he indicado, las circunstancias diferentes, no sólo la religiosa, sino por ejemplo el desarrollo más temprano de la revolución industrial, permitieron en países como Inglaterra la existencia de movimientos por la defensa de los derechos de la mujer y de movimientos feministas. Tampoco las feministas inglesas fueron en un principio muy propicias al control de natalidad, dedicándose a luchar por otros derechos de la mujer. Pero así se iba conformando el sentimiento de "persona" separado del de madre. En Inglaterra, además, el acceso a la cultura permitió que mujeres con alta preparación pudieran preocuparse por problemas de la mujer, como sucedió con Mary Carmichel Stopes.

La ciencia biológica que fue surgiendo en el siglo XIX, los estudios antropológicos aparentemente cargados de datos y comprobaciones científicas, vinieron a poner una nueva carga de determinismo sobre la mujer, pues parecía comprobarse su único valor como hembra reproductora y su inferioridad en todo otro aspecto. Los datos antropológicos parecían demostrar que la mujer era intelectualmente inferior al hombre, puesto que tenía una cabeza más pequeña y unas constantes vitales menores. Se producen durante las primeras décadas del siglo XX abiertas descalificaciones de la mujer en cuanto a su capacidad 
de trabajo físico e intelectual. Y cuando no es así, se adorna la cuestión, se disfraza con lo que ha sido la actitud más habitual en España; la postura paternalista.

Marañón nos da, en sus escritos, excelentes ejemplos de lo que pensaba un intelectual católico, liberal y bienpensante y además médico, de la mujer:

"La diferenciación de los dos sexos se había estudiado, hasta hace poco tiempo, tan sólo en su aspecto morfológico. Bien conocidos son, por ser del uso diario de los sentidos su apreciación, los caracteres sexuales primarios y secundarios que en la especie humana y en todo el reino animal separan al macho de la hembra. Los naturalistas y biólogos afinaron después las diferencias que a la afectividad imprime el sexo; es decir, el distinto modo de sentir y reaccionar en los momentos pasionales el alma de la mujer y la del hombre, distinción tan bien apreciada por el vulgo, que dice, por ejemplo, de un hombre pusilánime que "llora como una mujer", o que llama "varonil" a una mujer decidida. Pero no se reducen a esto las diferencias que el sexo imprime en la naturaleza humana. Los estudios recientes demuestran que el funcionamiento de cada célula de los diversos tejidos que constituyen el organismo es diferente en el varón y en la hembra; de ello resulta que es también diferente el conjunto de las misteriosas funciones de la transformación, aprovechamiento y eliminación de los materiales nutritivos que se conocen con el nombre general de "metabolimo orgánico". El metabolismo, lo más íntimo de la química del ser vivo, es, por lo tanto, perfectamente distinto en cada sexo. Blair Bell, es un sugestivo libro (The Sex Complex, 1916) insiste mucho en estos hechos y en la importancia que tienen para la comprensión del feminismo actual. El metabolismo del varón tiende a la transformación rápida, al gasto dispendioso de los materiales nutritivos; es catabólico, según la acertada expresión de Geddes y Thompson (The Evolution of Sexes, 1901); el de la hembra tiende a la síntesis, a la reserva; es anabólico; el primero es derroche; el segundo economía. He aqui ya marcada, y en lo más hondo de la vida del organismo, una diferencia que nos enseña, con la fría exactitud demostrativa de la físicoquímica, cuáles son los caminos divergentes que para cada sexo ha trazado el Destino. El hombre tiene construida su economía para el desgaste; es decir para la lucha en el ambiente externo. La mujer está hecha para el ahorro de energia, para concentrarla en si, no para dispersarla en torno; como que en su seno se ha de formar el hijo que prolongue su vida, y de su seno ha de brotar el alimento de los primeros tiempos del nuevo ser» (15). 
Este texto de Marañón demuestra el tipo de argumentación pseudocientífica que se utilizaba frecuentemente en las primeras décadas del siglo para apoyar y fundamentar lo que en realidad eran preconceptos y posturas ideológicas. Digo argumentos pseudocientíficos porque se mezclan conocimientos verdaderamente científicos, como la existencia del proceso metabólico, con extrapolaciones gratuitas y en absoluto comprobadas y en absoluto ciertas sobre un predominio del catabolismo sobre el anabolismo en el hombre, dando además a estos procesos del metabolismo una capacidad de determinación del comportamiento que no tiene ninguna base científica. Pero es que además, incluso si tales diferencias fueran ciertas no deberían en ningún caso ser determinantes del papel que cada ser humano quiera jugar en la vida.

Pero todo este tipo de argumentación tenía gran fuerza y contribuía a la presión que la sociedad ejercía sobre la mujer. Es evidente que la mujer española, la mujer de la clase media con ciertos contactos con el mundo del trabajo o de la cultura, la mayor parte de las veces se autodescalificaba en cuanto a su propia capacidad para desenvolverse en alguno de estos ámbitos. Así fueron de tardíos los movimientos feministas y de escasas y cortas sus reivindicaciones. Y, además, de politizadas o polarizadas sus posiciones. En el sentido de que, o se tenían unas ideas muy radicales, libertarias, o se caía dentro de las mismas concepciones sobre la maternidad y buena crianza de los hijos como tema primordial de toda reivindicación. En realidad, incluso los libertarios estaban imbuidos de estos conceptos. La mujer era una compañera, y tenía derecho a desenvolverse como quisiera y a tener actividades propias, pero sin chocar con el deber de ser madre, que estaba por encima de todo. No podemos entrar aquí en el análisis de la situación existente dentro de las diferentes clases sociales y grupos ideológicos. Pero lo cierto es que el contexto social daba muy pocas posibilidades a la mujer de que se sintiera con fuerza o con derecho a hacer reclamaciones en un ámbito que se saliera de sus derechos como madre y esposa.

\section{Los métodos anticonceptivos y la mujer}

Pensamos, pues, que es importante tener en cuenta esta situación social y conceptual de la mujer si queremos estudiar su actitud frente al sexo, la procreación y concretamente el uso de métodos anticoncep- 
tivos. Y debemos también considerar que el conocimiento de métodos para evitar la concepción debe analizarse por lo menos en cuanto a su posibilidad de transmisión por dos canales: uno, la comunicación personal, familiar; la otra, la comunicación escrita, la información a nivel profesional o intelectual.

La comunicación personal, entre familiares, vecinas, comadronas o curanderos es enormemente difícil de conocer. Se trata de cuestiones íntimas, prohibidas, penadas, que deben mantenerse en secreto.

En Gran Bretaña y también en Francia se han hecho estudios sobre esta vía de transmisión de conocimientos y sobre el uso de técnicas anticonceptivas; no sólo sobre el coitus interruptus, ampliamente utilizado en todos los países, incluida España (nos los indica la fuerte reacción condenatoria de la iglesia y la realidad de la existencia de un cierto control de natalidad), sino del uso de esponjas, diafragmas, duchas vaginales y diversas sustancias químicas. También se sabe que se utilizaban métodos de abstinencia parcial, ligada al ciclo ovulatorio. El problema es que, como el ciclo menstrual y el momento de la ovulación no se conocieron realmente hasta la década de los años veinte (Ogino publicó sus trabajos en 1923), y se creía que el período no fértil era justamente el que tiene más probabilidades de que se produzca la ovulación, el método no era en absoluto efectivo, más bien todo lo contrario.

En España hay poquísimos datos sobre los métodos caseros. No sabemos, ni tenemos idea que nos permita valorar de alguna manera en que proporción se utilizaban. Sólo las indicaciones que nos pueden dar los estudios de natalidad que demuestran que se produjo descenso de natalidad, sobre todo, como sucedía también en otros países, en las clases medias.

Una encuesta realizada por el Ateneo a lo largo y ancho de todo el país y muy minuciosa, encuesta que se desarrolló entre 1900 y 1914 (16), presenta respuestas sobre este tema de una sola provincia española, Santa Cruz de Tenerife. Bajo el Título «Práctica para no ser fecundada» dice:

«1. Tomar un vaso de agua antes del coito.

2. Tomar un vaso de agua en ayunas con polvos de alcanfor.

3. Tomar, a la mañana siguiente del coito, una taza de culandrillo.

4. Beber una infusión de alquilara o servilleta o chupar la raíz.

5. Cubrir la mujer al hombre.»

En cuanto a la comunicación escrita o a nivel profesional, debemos decir que fue enormemente escasa. La información sobre técnicas an- 
ticonceptivas se produjo, en general, a partir de grupos minoritarios, como la mencionada Liga de Regeneración Humana y la revista Salud $y$ Fuerza y sus publicaciones y conferencias, todo ello a comienzos de siglo (17). En El Socialista aparecía publicidad de preservativos, pero no llegaba a publicar sistemáticamente artículos sobre el tema. Debemos decir, además, que anarquistas y socialistas, aunque apoyaban las reivindicaciones feministas y las luchas - al nivel modesto que existían- por los derechos de la mujer, no eran grandes partidarios del control de natalidad. Por lo menos no fue así hasta casi entrados los años treinta, en que algunas figuras, en general abogados -como Jiménez de Asúa o Noguera, o pedagogos como Luis Huerta- pero rara vez médicos, comenzaron a plantear el control de natalidad como medio de evitar males mayores: como el aborto o el infanticidio (muy abundantes ambos) e incluso como defensa de la libertad y dignidad de la mujer. Creemos que fue una postura minoritaria. En los años treinta el papel más importante con respecto a la difusión de los temas relacionados con la sexualidad y la procreación lo tuvo la mencionada Liga para la Reforma sexual sobre bases científicas y su revista, Sexus (18).

En este punto es necesario señalar que, tanto las posibilidades de acceso al conocimiento, como la reacción de la mujer ante el problema debían estar necesariamente condicionadas - con las siempre posibles excepciones individuales-, por la clase a la que pertenecían y por el medio, urbano o campesino, en el que se desenvolvían. Y también, claro está, por la condición de casada o soltera. Me explico: una mujer de clase media alta, aunque quizás más presionada por modos, costumbres y normas en unos aspectos, estaba, o podía estar, algo más en contacto con el conocimiento científico, con la cultura en general, que una mujera obrera. También dada su condición más elevada, es posible que fuera objeto de más consejos e informaciones, aunque fueran veladas, tanto de médicos como de religiosos. Lo cierto es que la natalidad de las clases media y alta va descendiendo con los años, como indica en su estudio Severino Aznar (19).

Las mujeres de clase obrera, urbanas, tenían, indudablemente, mucho menor contacto con la cultura. Su información provendría de otras mujeres o de curanderos o comadronas. Y muchas veces sería, claro está, falsa, así como lo eran la mayoría de los productos para abortar. Los hijos podían ser más brazos, pero en momentos de crisis eran sólo más bocas. Los abortos e infanticidios y el abandono, en la inclusa o no, eran abundantísimos. Y no digamos ya en el caso de las madres solteras, rechazadas totalmente incluso por instituciones de caridad. 
Las mujeres campesinas casadas quizás tuvieran menos interés en controlar la natalidad, ya que el aumento de brazos podía ser más importante que lo que significaba un aumento de bocas. Sin embargo, en épocas de agudización de la crisis, como sucedió tanto a finales del siglo XIX como después, alrededor del año veinte y alrededor del treinta, lo que solía contar era el peso de las muchas bocas.

La mujer, pues, además de pertenecer a una clase social pertenecía a una sociedad dada, la española, con sus particulares características, entre ellas una fuerte moral tradicional y una Iglesia que a lo largo del siglo XX va aumentando su organización y poder, manteniendo las posturas más tradicionales y conservadoras que darse pudieran dentro de la propia iglesia. Iglesia que, además, fue motor de la organización de algunos grupos "feministas», que planteaban sus reivindicaciones siempre dentro de lo que fuera mejor para sus papeles de madre y esposa. La mujer, insistimos, además de estar fuertemente condicionada por toda esta estructura ideológica, y de pertenecer a un determinado nivel social que también la condiciona, es considerada, gracias a la biología y a la ciencia, como un ser inferior al hombre.

\section{La medicina y los médicos}

Pensamos que los médicos españoles contribuyeron en gran medida tanto a la falta de información correcta de las mujeres con respecto a los métodos anticonceptivos, como a la consideración de éstas como seres biológicamente inferiores al hombre en cuanto al intelecto, a su capacidad para desarrollar actividades intelectuales y profesionales. Indudablemente no todos los médicos tenían la misma actitud, pero la revisión de publicaciones, alguna tan representativa como El Siglo Médico, demuestra que ni siquiera a nivel profesional se informaba sobre el control de natalidad y los posibles métodos anticonceptivos. Ni siquiera se constata, en la publicación antes citada, la valoración de lo que significó el descubrimiento del ciclo menstrual y la propuesta de Ogino para controlar la natalidad. Tampoco a finales de los años veinte se ven artículos sobre este tema, a no ser en publicaciones especializadas, tanto libros como en la mencionada revista Sexus. Sin embargo, se produce en esos años, finales de los veinte, una amplia movilización a favor de la educación sexual, de la eugenesia, etc. Sólo en este terreno, el de la eugenesia, se oyen voces que hablan del control de la natalidad como medida útil y casi imprescindible para evitar la alta tasa de 
mortalidad infantil, el aborto y el infanticidio. Pero las que se oyen no son precisamente voces de médicos. En el Primer Curso Eugénico Español (20), suspendido por la Dictadura de Primo de Rivera - gracias a las fuertes presiones de la Iglesia y la burguesía bienpensante- por su llamado carácter "pornográfico», los que defendieron el control de natalidad y la anticoncepción fueron los abogados Jiménez de Asúa y Joaquín Noguera, y no los médicos Recaséns, Estella o Sanchís Banús. Tampoco Marañón, -que no llegó a pronunciar su conferencia en este curso por la citada suspensión- propuso nunca ninguna medida de control de natalidad que no fuera la abstinencia, aceptada por la iglesia.

En cuanto al tema de la inferioridad biológica de la mujer, el mayor conocimiento científico que van adquiriendo nuestros médicos a lo largo de las primeras décadas del siglo, y lo más ajustado de sus conocimientos, hicieron que en cierta medida perdiera fuerza el concepto de «inferioridad» en su sentido total y absoluto. Quieras que no, la mujer había demostrado en muchos casos que sí tenía capacidad para el trabajo intelectual, y la realidad hacía evidente, en aquellos años veinte del cambio en el vestir, del pelo a la "garçon" y del automóvil, que la tal inferioridad femenina era muy relativa. Se comienza a hablar entonces no de «inferioridad» sino de «especificidad» biológica. La mujer, se dice, no es inferior sino diferente, hecha por sobre todas las cosas para los altos fines de la maternidad, que se da a entender es casi incompatible, a la hora de la verdad, con otros fines. No es que fuera incompatible, ni mucho menos, con una buena educación de la mujer, pues esto contribuiría a que fuera mejor madre y más completa. Pero siempre que todo aprendizaje o actividad estuviesen subordinados a la función de madre y contribuyesen a mejorarla. La profesionalización parecía ser el aspecto más incompatible con tal fundamental lahor. Claro que obreras y campesinas, empleadas de servicio o lavanderas, seguían trabajando duramente sin grandes protestas por parte de los bienpensantes. Era penoso, pero no parecía tan reprobable como que una madre burguesa abandonase unas horas a sus retoños para acudir a una oficina. Evidentemente había sectores de la sociedad que no estaban de acuerdo con esta postura de marginación de la mujer, pero en mayor o menor medida se aceptaba — cuando se podía, clarola subordinación de toda actividad al papel de madre.

De todo es conocida la enorme importancia de Marañón y de su pensamiento en el ambiente cultural español de los años veinte y treinta. Su prestigio era enorme, tanto entre los médicos como a nivel popular entre las clases medias y la juventud universitaria. Según recuerda mi 
madre, el libro de Marañón Amor, conveniencia y eugenesia, publicado en los años treinta, fue enormemente popular entre los jóvenes de las clases medias y entre los universitarios. Por esta razón tomaremos a Marañón como ejemplo de las ideas de un médico, digamos que no especialmente conservador, de talante liberal y muy considerado social y profesionalmente.

Dice Marañón en «Biología y Feminismo», su conferencia de 1920:

«CALIDAD DEL RENDIMIENTO INTELECTUAL DE LA MUJER

Otras varias razones, ya más conocidas hablan también en apoyo de la tesis de que las actividades que exigen un esfuerzo intelectual original son extrañas a la psicología normal del sexo femenino. Es una de ellas el escaso número de mujeres que han sobresalido en el mundo intelectual.»

Piensa que algunas mujeres sobresalientes ha habido, pero:

"tenemos que reconocer que al talento femenino, en general, aunque alcance límites avanzados de claridad y penetración, le falta originalidad. Por eso en la ciencia las mujeres son buenas técnicas, pero no inventoras; y en el arte buenas ejecutantes, intérpretes y copistas, pero no suelen innovar nada.»

Y continúa, para que se vea lo equilibrado de su posición:

«A esto arguyen las feministas que el menor rendimiento intelectual del sexo femenino se debe a que, ocupada la mujer en las labores caseras, no ha podido perfeccionar el desarrollo anatómico y funcional de su cerebro."

Pero no es este el problema, arguye Marañon:

«Lo que en la mujer se opone al predominio de las funciones intelectuales no es un inferioridad - funcional o anatómica- de su sistema nervioso -en la cual yo no creo-, es simplemente su sexo, que indefectiblemente marca y marcará siempre otros rumbos a sus actividades. "¡Cuando se dispense a las mujeres más inteligentes de la preocupación de la prole! ¡Ah, no, maestro!" (Se refiere a una opinión de Cajal) Las más inteligentes, precisamente por serlo, si son mujeres normales, no aceptarán esa dispensa, no cambiarán por todo el rendimiento de gloria que da el ejercicio social del intelecto, la pura y escondida alegría de ser madres por entero, sin restar un segundo al vulgar pero inefable "cuidado de la prole".» 
El razonamiento es evidentemente maquiavélico. Parte de una aparente postura de respeto y defensa de la mujer. No hay inferioridad cerebral, pero ¡hay sexo! y eso es suficiente. Si una mujer es inteligente y normal, nos dice, siempre.preferirá, por encima de todo, el cuidado de la prole, que se hace incompatible con otra actividad que robaría tiempo a la más importante. Se deduce que una mujer que no siente y actúa así será poco inteligente y anormal. Veamos lo que dice a continuación nuestra gran autoridad:

«ANORMALIDAD SEXUAL DE LAS MUJERES GENIALES.

Y aún queda la razón más fuerte en pro del carácter heterosexual de la actuación social de la mujer. Y es, que muchas de esas mujeres que justamente han alcanzado la celebridad, en el terreno en que la alcanzan los hombres, han sido poco mujeres, han tenido en sus rasgos físicos, en su sensibilidad, en su mentalidad tonos marcadamente masculinos.» (...) «Y téngase en cuenta que esta tendencia puede coexistir con una perfecta maternidad, (¿ en qué quedamos, pues?), como ocurría - y lo cito por ser un ejemplo tal vez el más excelso y respetable- en nuestra doña Concepción Arenal, que junto con el corazón más femenino de su tiempo, poseía un cerebro enteramente varonil, vistiendo a veces la indumentaria de nuestro sexo, y adoptando tocados y actitudes, dentro de su nobleza, muy de hombre, como puede verse en los retratos que de ella se conservan. Un poco de verdad hay, pues, en el concepto de Moebius, cuando afirma que "la mujer científica o artista es un producto de degeneración".»

Y remata Marañón este prodigio de argumentación de esta manera:

«La mujer normal, por consiguiente, en nuestros tiempos, como en los antiguos, tiene y tendrá siempre, como misión fundamental, el ejercicio de las funciones sexuales primarias que constituyen la maternidad. Las leyes biológicas son invariables; están por encima de toda discusión literaria y filosófica; y estas leyes marcan, con inequívoca certeza, la verdad que acabamos de enunciar» (21).

Independientemente de que sea falsa la existencia de unas leyes biológicas que apoyen las afirmaciones de Marañón, su argumentación es totalmente circular, de manera que siempre será imposible demostrar que una mujer y por tanto las mujeres pueden realizar trabajos intelectuales válidos, e incluso hacerlos perfectamente compatibles con una buena maternidad, si cada vez que se constata tal situación se dice que tal mujer es anormal. Por lo tanto todo lo que hace Marañón es afir- 
mar algo de lo que él está convencido, puesto que piensa que las hormonas son determinantes del comportamiento hasta en sus más nimios detalles y, además, hace incompatibles la función de pensar con la de tener hijos. Pensemos lo grave de tales argumentos situándonos en aquella época. Que una figura tan significativa dijera tales cosas, en tono protector y paternalista, y lanzando toda la ciencia y las leyes de la biología encima de la cabeza de las pobres mujeres, debía obtener como resultado el que muy pocas se atrevieran a contradecir tales argumentaciones, so pena de ser tachadas de anormales.

En cuanto al control de natalidad, ya hemos dicho que la postura más frecuente de los médicos fue la de abstenerse de opinar. Ni los médicos eugenistas de comienzo de siglo, ni los posteriores defensores de la educación sexual, de la maternidad consciente y de, incluso, la esterilización de anormales, se refieren a los métodos anticonceptivos. En realidad es la misma postura que adoptaron los médicos ingleses en el siglo XIX.

Revisando las ponencias presentadas en el año 1933 a las Primeras Jornadas Eugénicas Españolas (22), en las que participaron unos 50 intelectuales, vemos que de más de veinte médicos participantes hay sólo cinco que se refieran al control de natalidad. Y era una reunión de intelectuales progresistas.

Yagüe y Espinosa, secretario de la Sociedad Española de Higiene, habla del certificado prenupcial y del problemaa de las uniones fuera del matrimonio; hecho este, que, dice, es muy frecuente que se produzca en todas las clases sociales: «las más de las veces con prácticas neomalthusianas que evitan engañosamente el deshonor». Opinión, por tanto, en contra de tales práctica «neomalthusianas».

Francisco Haro, reputado tocólogo y ginecólogo y veterano y entusiasta eugenista (23), en su comunicación "Concepción y Anticoncepción» dice que las indicaciones de los «anticoncepcionales» son: enfermedad o taras, razones económicas, de higiene, o razones particulares y sentimentales. Señala Francisco Haro que los anticonceptivos deben utilizarse en circunstancias muy especiales, pero da la clasificación de ellos establecida por Mary Carmichel Stopes y también explicaciones detalladas sobre su uso. Apoya, pues, el uso de métodos para controlar la natalidad.

José María Otaola, tocólogo y ginecólogo dice, también en «Concepción y Anticoncepción »: "Que algunos pretendan privadamente evitar la concepción con su propia responsabilidad, es un derecho individual 
discutible según el punto de vista desde el que se enfoque la cuestión; pero si la procreación ilimitada se extiende a los inadaptados, la cuestión toma otro aspecto...» Otaola, ambiguo en cuanto al derecho individual al control de natalidad, es, sin embargo, partidario de la esterilización cuando de inadaptados sociales se trata.

Jimena Fernández de la Vega en «La herencia biológica en el hombre», además de indicar que en las capas sociales con mayor personalidad, la natalidad estaba disminuyendo en proporciones extraordinarias, por limitación voluntaria de hijos y por disminución en el número de matrimonios, dice: «Racialmente sólo tiene interés aquella medida social que tiende a evitar el aumento de los imbéciles y de los criminales y sólo estos...", o sea que racialmente sólo interesaba la esterilización. Sin embargo, agrega, si se limita la natalidad y se disminuye la mortalidad se eliminan las posibilidades de selección natural y se para el proceso evolutivo. Desde este punto de vista,.de aplicación de la selección natural a la sociedad, Jimena Fernández de la Vega, llevada del cientificismo de la época, ve como negativo el control de natalidad (24).

El único que es claro y transparente en su conferencia sobre «Pedagogía sexual» es Gonzalo Rodríguez Lafora. Dice que en la educación sexual postpuberal deben reflejarse dos aspectos, uno filosófico y otro médico, higiénico y eugénico. «El aspecto médico se orienta hacia la prevención de las enfermedades sexuales, la generación consciente y eugénica, la prevención de la concepción y otros aspectos del problema.» Piensa Lafora que en la moderna educación sexual son muy importantes los «medios anticoncepcionales», a los que Bernard Shaw ha denominado «el gran invento del siglo». Pero, observa el autor, el aspecto negativo que presentan estos métodos es que, como siempre, todo recae sobre la mujer.

Lafora, con su excelente formación científica y su claridad al hablar.de tan escabroso tema, es una excepción entre los médicos. La.postura de los médicos en general, de la corporación médica, fue, como hemos dicho repetidamente, de oscurantismo. Pensamos que esta postura negativa y regresiva que suele mantener la corporación médica tiene nefastas repercusiones sociales. El oscurantismo que siempre han mantenido sobre los temas sexuales han contribuido a la desinformación, las prácticas abortivas no controladas, la mala higiene y tratamiento de este tipo de problemas; y ha contribuido, claro está, a mantener a la mujer en una situación de culpa por un lado y de indefensión por otro. Angus McLaren (Ver nota 4), que ha estudiado el comporta- 
miento de los médicos en Gran Bretaña con respecto a esta cuestión, considera que estos profesionales sentían que la contracepción era, al quedar la decisión y la realización en manos del matrimonio, y peor aún, en manos de la mujer, un ataque a su control profesional, a su papel también de árbitros morales, y a su papel de machos. No debemos olvidar cuál era la tónica general imperante en cuanto a la consideración de la mujer, que hemos visto a través de los escritos de Marañón. Es interesante destacar cómo abogados, pedagogos, publicistas e incluso teólogos progresistas, participantes también en las Jornadas Eugénicas, fueron mucho más claros en sus posturas no sólo con respecto al control de natalidad sino también con respecto al papel de la mujer, frente a la cual manifiestan posturas mucho más progresistas que los médicos. Así puede comprobarse en los escritos de Jiménez de Asúa, Joaquín Noguera, Luis Huerta o Hildegart.

Como hemos dicho, pues, pensamos que la figura que más podía haber contribuido a mejorar el nivel sanitario, de información sexual, evitando que muchas mujeres cayeran en manos de comadronas sin escrúpulos, de curanderos o de estafadores vendedores de productos abortivos, anticonceptivos, etc., y contribuyendo a un mejor dominio de la mujer sobre su propio cuerpo, el médico, no sólo no cumplió con tan hermoso papel sino que fue, frente a otros profesionales, una fuerza regresiva, de freno de la difusión del conocimiento.

En resumen, pues, las mujeres españolas tuvieron escaso conocimiento, y malo, de los posibles métodos anticonceptivos, incluido el método Ogino. Abundaron las altas tasas de natalidad y también la alta mortalidad infantil, el aborto clandestino y el infanticidio. Sin duda, la pertenencia a determinadas clases sociales permitió el acceso a ciertos conocimientos sobre los métodos anticonceptivos, ya que las clases medias y altas manifiestan en los años veinte y treinta un claro descenso en la tasa de natalidad.

\section{NOTAS}

(1) Marañon, G. (1920): "Biología y Feminismo», Conferencia pronunciada en la Sociedad Económica de Amigos del País, Sevilla, 21 de febrero de 1920. Publicado en El Siglo Médico, núms. del 10, 13, 27 de marzo y 10 de abril de 1920.

(2) Hildegart Rodriguez Carballeira es conocida más que nada por su historia personal. Criada y educada por su madre para ser una niña prodigio, fue, según parece, asesinada por ella cuando contaba aún muy pocos años. Hildegart, abogado y publicista se dedicó a los temas de control de natalidad y educación sexual, participandó en múltiples campañas sanitarias. Publicó entre otras cosas: El problema eugénico, punto de vista de una mujer moderna, Madrid, 1930, Educación Sexual, Madrid, 1931, Profilaxis an- 
ticoncepcional, Valencia, 1931, etc. Su ponencia en las Jornadas Eugénicas fue: «Maternidad consciente», que comprendía los siguientes apartados: I.-Malthusiano y neomalthusiano. II.-Vista panorámica del «Birth Control». III.-Estudio científico de la anticoncepción, y IV.-Repercusiones jurídicas.

(3) Malthus, R.: An Essay on the Principle of Population, as it Affects the Future Improvement of Society with Remarks on the Speculation of Mr. Godwin, Mr. Condorcet and other Writers. La primera edición es de 1798, pero hubo muchas más hasta bien entrado el siglo xIx. En castellano: Primer Ensayo sobre la Población, Madrid, 1966.

(4) McLAREN, Angus (1978): Birth-control in nineteenth century England. Londres.

(5) CONDORCET, A. N. (1975): Esquisse d'un tableau historique des progrès de l'esprit humain. Paris. Esta obra, optimista en cuanto a los buenos resultados del progreso, fue muy popular en Gran Bretaña, y por ello contestada por Malthus.

(6) El Utilitarismo, doctrina moral y política que predicaba la búsqueda de la mayor felicidad para el mayor número, recibió su forma teórica más desarrollada y completa de manos de J. Bentham (1748-1832). Entre otros aspectos, buscaban el perfeccionamiento del hombre a través del progreso, y siempre tomando como medida de todo la búsqueda de la felicidad.

(7) EyLER, J. M. (1979): Victorian Social Medicine. The Ideas and Methods of William Farr. Londres.

(8) La gran labor realizada por Florence Nightingale fue, justamente, establecer condiciones higiénicas y organizar las enfermerías, organizando posteriormente un cuerpo de enfermeras profesionales que comprendieran la importancia de tales medidas. Su acción fue fundamental para salvar vidas de heridos en la guerra de Crimea.

(9) PAUL RoBIN (1837-1919), pedagogo neomalthusiano, abandona las alternativas revolucionarias de su época para, influido por el positivismo, buscar un camino de reforma del ser humano. La regeneración del hombre girará en torno al problema de la superpoblación de la tierra y a la desigualdad intelectual existente entre los individuos. Afirmará que la solución de la cuestión social depende de las medidas que se arbitren para frenar el crecimiento de la humanidad. Se preocupó, además, por la enseñanza integral, preocupación e interés que compartió Ferrer i Guardia. Buen nacimiento, buena educación, buena organización social serían los puntos centrales de la búsqueda de un nuevo orden social. Robin fundó la Liga de Regeneración Humana en 1895, planteando como uno de los puntos esenciales el neomalthusianismo. No fue apoyado ni por los socialistas ni por los anarquistas, que no estaban de acuerdo con el neomalthusianismo. La Liga se mantuvo hasta 1908.

(10) En La Revista Blanca del 15 de diciembre de 1904, p. 383, se dice: «Ha aparecido el primer número de una revista mensual, órgano de la sección española de la Liga de Regeneración Humana. Se dedica a propagar la procreación consciente y limitada de la especie humana. El criterio que sustenta dicha revista en el terreno político y religioso es anarquista y ateo; en el terreno económico, cree que la procreación abundante y natural es un mal para la misma especie y hay que limitarla, según esos neomalthusianistas, en bien de todos. Nuestro criterio en este punto, es que el anarquismo malthusianista es un anarquismo que ha producido el modo de ser de la familia y de la producción actual, que no son, por cierto anarquistas.»

(11) En 1883, en su libro Inquiries into Human Faculties, decía Galton que su intención era «mencionar varios tópicos más o menos conectados con aquello del cultivo de la raza, o, como podemos llamarlo, con las cuestiones eugénicas. Esto es, con problemas relacionados con lo que se llama en griego eugenes, es decir, de buen linaje, dotado hereditariamente con buena cualidades.» 
En 1904, cuando Galton propone a la Universidad de Londres el montaje de un Laboratorio de Eugénica, se forma un comité, en el que también él participa, que elabora una definición de eugenesia que será la que en adelante se difunda: «La expresión Eugénica Nacional se define aquí como el estudio de los medios que están bajo control social que pueden beneficiar o perjudicar las cualidades raciales de las generaciones futuras, tanto física como mentalmente.»

(12) La comunicación que presentó Ignacio Valentí y Vivó al Primer Congreso Internacional de Eugenesia se titulaba The History of a Healthy Sane Family, showing Longevity, in Catalonia."

(13) A finales del siglo pasado y comienzos de este, la crisis hizo surgir lo que se llamó movimiento regeneracionista. Se consideraba que existía una situación de decadencia nacional. La raza y el estado en el que se encontraba el pueblo español se consideraban parte fundamental de ese fenómeno. Hubo protestas por esta actitud como la de Jaime Vera, que dice: «Bajeza es adular al pueblo. Injusticia insigne cargarle las culpas por los mismos que las cometieron y siguen cometiéndolas. Hasta hay quien declara al pueblo español incapaz de remedio por condiciones étnicas y antropológicas, por su cráneo y su cerebro.» El Socialista, 1 de mayo de 1900.

(14) Un salmantino, Federico Gómez-Arias, estableció en su provincia un premio anual de mil pesetas «a una señorita de Salamanca de buena constitución física, robustez, salud, belleza, buena conducta, instrucción elemental al menos y edad de quince a veintitrés, que vaya a unirse canónica o civilmente a un hombre de análogas condiciones físicas y morales y cuya edad sea acomodada a la de la novia ». Publicado por Andrenio en Nuevo Mundo, núm. 874 del 6 de octubre de 1910

(15) Marañón, G. (1920): Biología y Feminismo, p. 11.

(16) Encuesta del Ateneo 1900-1914. Museo Etnológico-Manuscrito.

(17) Salud y fuerza Revista Mensual Ilustrada de la Liga de Regeneración Humana, cuyo fundador fue, en 1904, Luis Bulffi, contaba además con una "Biblioteca» en que ofrecía obras como Exposición de Doctrinas Neo-malthusianas y ${ }_{i} H u e l g a$ de Vientres! del mismo Bulffi, El problema de la población de S. Faure, Inmoralidad del matrimonio, La préservation sexuelle, Le Bréviaire de la femme enceinte etc. En los anuncios de la Revista se podía leer: «Conos Preservativos del embarazo y de las enfermedades sexuales, del Dr. Mascaux»; «Formolodor Veignault, Esterilizante el más poderoso»; «El Obturador Uterino, evita los embarazos contraindicados", etc..

(18) La rama española de la Liga para la Reforma Sexual sobre Bases científicas se fundó en 1932. Su primer presidente fue Marañón, pero en 1933 su presidente era Juan Noguera. La secretaría seguía siendo Hildegart. La Liga publicaba la revista Sexus, que tenía también a Hildegart como secretaria, y cuyo Comité de Redacción estaba compuesto, en su primer número, de octubre-noviembre de 1932 por Vital Aza, F. Haro, Luis Huerta, César Juarros, Luis Jiménez de Asúa, Gregorio Marañón, José María Otaola, Mariano Ruiz Funes y José Sánchez Covisa.

(19) AzNAR, Severino (1929): «El promedio diferencial de la reproductividad de las clases sociales de Madrid", Boletín de la Universidad de Madrid, enero de 1929, núm. 1.

(20) El Primer Curso Eugénico Español, organizado por Gaceta Médica Española y al que se adhirieron la Sociedad Española de Biología, la Sociedad Española de Antropología, la Sociedad Ginecológica Española y el Colegio de Doctores de Madrid. Estaban programadas 10 conferencias, de febrero hasta abril de 1928. Sólo se realizaron cinco: "Eugenesia y procreación» por Sebastián Recaséns; «Aspecto jurídico de la maternidad consciente» por Jiménez de Asúa; «Los niños que vemos en nuestros hospitales» por el Dr. J. Estella; «La maternidad y el infanticidio ante el derecho» por Joaquín Noguera 
y «Neuropatías producidas o influenciadas por la procreación excesiva o patológica en la pobreza del medio» por el Dr. Sanchís Banús. El Curso fue suspendido por Real Decreto firmado el 17 de marzo de 1928, pues se consideraba que se trataban temas que no debían ser escuchados por el público en general. Lo cierto es que las conferencias se realizaban con el anfiteatro de la Facultad de Medicina totalmente lleno, lo que demuestra el enorme interés que despertaban estos temas.

(21) Marañon, G.: Biología y Feminismo, pp. 16 y 17.

(22) Las Primeras Jornadas Eugénicas Españolas se realizaron en Madrid en abril y mayo de 1933. Agruparon a gran cantidad de científicos e intelectuales que hablaron sobre temas de sexualidad, amor, maternidad, genética, etc. Las Jornadas están publicadas en dos volúmenes con el nombre de Genética, Eugenesia y Pedagogía Sexual, y tienen como directores de la obra a E. Noguera y Luis Huerta: Javier Morata Editor, Madrid, 1934.

(23) Francisco Haro publicó un interesante libro sobre eugenesia, en el que además ofrece una somera visión histórica del tema en nuestro país: Eugenesia y Matrimonio, J. Morata, ed., Madrid, 1932.

(24) Jimena Fernández de la Vega, médico, fue pensionada por la Junta para Ampliación de Estudios para estudiar genética humana en Alemania, Austria e Italia. A pesar de ser de los pocos profesionales con conocimientos actualizados sobre el tema, prácticamente no pudo hacer más que dar algunos cursillos, que fueron luego publicados como libro. Tal La herencia fisiopatológica en la especie humana. Espasa Calpe, Madrid, 1935. En la Jornadas Eugénicas de 1933 participó con «La herencia biológica en el hombre: I. Herencia de los caracteres psicológicos. II. Selección y Contraselección», Vol. I de Genética, Eugenesia y Pedagogía Sexual, pp. 159 a 181. 